\title{
Assessing the Consistency of the Design of Iranian Websites with Cultural Characteristics of their Target Users: An Investigation of the Hofstede Model
}

\author{
${ }^{*}$ Maryamossadat Mousavi ${ }^{1}$, Datis Khajeheian ${ }^{2}$ \\ ${ }^{1}$ Multimedia University, Malaysia \\ ${ }^{2}$ University of Tehran, Tehran, Iran \\ *mrym.mousavi@gmail.com
}

\begin{abstract}
The influence of culture on the design of websites has been researched extensively. A group of researchers has implemented Hofstede's Cultural Dimensions as a proper framework to study this subject. The literature supports that cultural characteristics of the Hofstede model are significantly present in the design of websites. It also suggests that making a website compatible with the cultural characteristics of the target users facilitates effective interaction of the users with the websites. Building upon these observations, the present study has investigated the impact of Iranian culture on the design of Iranian university websites. We have measured the consistency of their design with the cultural characteristics of their target users and studied the possible improvements brought about by a culturally harmonious design. To this end, one sample t-test method is applied to compare the scores given by the end users for each cultural dimension, with the scores of the Hofstede model to measure the level of consistency. The results suggest that the design of Iranian university websites represents a more collectivistic and masculine culture with higher indices of power distance than what is specified in the Hofstede model and a change towards a more individualistic, elegant and informal design is necessary for the websites to improve their conformance to the cultural elements of Iranian users and improve their experience. To our knowledge, the present paper is novel in two aspects: firstly, it focuses on the case for Iran, which has not been extensively studied before; secondly, it makes a parallel study to measure the users' preferences and compares it to the predictions made by the Hofstede model.
\end{abstract}

Keywords: Website design, Cultural Dimensions, the Hofstede model, Iranian University Websites

\section{Introduction}

Motivation: Technology and culture are known to have a mutual and delicate interaction, which has been researched extensively. World Wide Web as an example of modern technologies has influenced local cultures around the world by introducing concepts such as e-commerce, e learning, e marketing and cyber law. In return, various aspects of web technology are affected by local cultures. For instance, the acceptance and development of web services in various countries is subject to perceptions and expectations of users with different cultural backgrounds. Among different aspects of web technology, the interface design of websites, as the prime points of interaction with users, is crucially important for a successful and effective presence in the cyber space. A well-designed website can bring about trust, satisfaction and high return rate of users. Hence, such a website can meet the goals of this medium more effectively, for instance more effective knowledge sharing, better communication and more successful marketing. Achieving these outcomes is impossible without considering the role of culture in preferences and perceptions of the target end-users. In the recent years researchers have explored different elements of interface design in various cultures and found that cultural characteristics are meaningfully reflected in the design of websites in different countries (cf. Nantel, 2008; Van Iwaarden et al., 2004; Lodge, 2007; Callahan, 2006; Marcus, 2000; Alostath \& Khalfan, 2007). They linked various design features such as colors, pictures, layout, information organization, and navigation with cultural characteristics of different countries and suggested that creating a compatible website with the cultural characteristics of the target users facilitates and improves effective communication with the website. Considering the cultural studies of website design, we find that some cultures (e.g., West Asian countries such as Iran) are not addressed in this set of collection and there is no empirical research available about them. They are also confined to a mere description of the cultural differences, without putting their results into a practical perspective and relating the design features to the user perception. Bearing in 
mind the above-mentioned gaps, the present study has focused on the Iranian culture and the design of Iranian state university websites as one of the less studied countries in the cultural studies of website design. In our study, we have used a relatively comprehensive collection of cultural indicators. We applied these indicators to measure if the design of the Iranian university websites follows the cultural characteristics of their target users, and what practical suggestions can be given in this regard to improve the cultural consistency of the Iranian websites' design.

Research Questions: In view of the purpose of this research, the following three questions have been put forward:

- What cultural characteristics (based on the Hofstede model) are reflected in the design of Iranian university websites?

- Are these characteristics consistent with Hofstede's scores about Iranian culture?

- What suggestions can be made in order to improve the usability of Iranian websites through a culturally harmonious design?

\section{Literature Review}

Researchers have implemented various methods and theories to explore the relationship between cultural factors and the preferences of website developers and website users. One of the most widely used cultural theories in the area of interface design belongs to Hofstede (1991). Despite all the criticisms and shortcomings expressed about the theory of Hofstede, this model has been extensively used as one of the theories by which we can compare and discuss cultural trends and preferences among various countries in the world.

Hofstede's Cultural Dimensions: during the years 1978-83, a Dutch anthropologist with the name of "Geert Hofstede" carried out a survey on the IBM staff members in 53 different countries around the word. Making use of this large data set, he determined four cultural dimensions, which explain the patterns of differences and similarities between various cultures around the word. The four cultural elements in Hofstede's model are as follows (Hofstede, 1991): Power Distance refers to the extent to which inequality in distribution of power is tolerable among the members of a society. Uncertainty Avoidance refers to the extent to which ambiguity and vagueness is tolerable in a particular culture. Masculinity versus Femininity points at the role of both genders in a society. Individualism versus collectivism stresses the strength of relationships or ties between people as members of different groups in a culture. In 1987, thanks to the cooperation of Hofstede with a Chinese scholar, a new element was added to this model, which was entitled as "long term versus short term orientation". The new dimension concerns attributes such as respect for tradition and the elderly, being patient and hard working and living a simple life that are more relevant to the culture of the Eastern countries.

Iranian Culture in Hofstede's Model: According to the analysis of Hofstede, for the Iranian culture uncertainty avoidance and power distance score the highest among the four dimensions with the ranking of 59 and 58 respectively. In addition Iranian culture is reflected to be a relatively collectivist culture with the score of 41 in individualism and takes a middle position on masculinity dimension with the score of 49. Although Iran is not at the extremes of the spectrum in any of the above-mentioned dimensions and is ranked almost in the middle, according to the overall picture built up by Hofstede's theory, Iranian culture is a culture with a relatively high uncertainty avoidance and power distance, with some collectivist characteristic and a medium ranking in masculinity/femininity.

Cultural studies of website design: As mentioned earlier, following the theory of Hofstede in recent years some researchers started to implement his cultural model to explore the preferred characteristics of website design in various cultures. Marcus who is a pioneer in this regard (2000, 2001, 2002 and 2009) has done a series of researches regarding the relationship between the dimensions of Hofstede and the features of website design. He examined and compared the design of websites from different countries particularly those ranked the highest and lowest in each cultural dimension and linked up the differences and similarities in their design to the cultural dimensions of Hofstede. He noticed that cultural characteristics are reflected in the design of websites in different countries. Robbins and Stylianou $(2002,2008)$ also investigated the design 
of a group of large global corporate websites within the context Hofstede's model. They carried out their research two times with the interval of ten years to see if there are any changes in the national cultures over a this period of time. They realized that although cultural differences in the design of websites are still relevant and need to be considered by website developers, they have become lesser and there is a smooth movement towards homogeneity in the design of websites. Ahmed, Mouratidis \& Preston (2008) compared Malaysian and British websites from different genres based on two of Hofstede's cultural dimensions, power distance and individualism/collectivism claiming that these two dimensions are more significant in differentiation between Western and Asian cultures. They findings reveal significant cultural differences in design of the content and appearance of the two countries local websites. Callahan (2006) also compared the organization of information, graphical and visual design of university websites from Malaysia, Austria, the United States, Ecuador, Japan, Sweden, Greece and Denmark in the context of Hofstede model. She found the design elements of the websites, relatively similar within a particular culture, varied across different countries and quite in line with the cultural dimensions of Hofstede. However, the scores calculated for each element were not as strong as they were predicted in Hofstede's model. In parallel to the studies comparing the design elements of websites to bring out the cultural differences, some researchers attempted to find the cultural variations based on the preferences of website users. For example, Cyr (2008) examined the perception of users from eight countries to find the differences in information, navigation and visual website design preferences. His findings, along the same line of many other past researches, show meaningful variations in preferred design features across cultures particularly between European and North American cluster (representing by USA, Canada, Germany) with Confucian Asian (representing by Japan). In all of the aforementioned studies, researchers found and introduced some interface design characteristics that represent cultural attributes of the Hofstede model. The following table summarizes these cultural indicators and divides them into four groups based on the four cultural dimensions of Hofstede's model.

\section{Indicators of High Power Distance in Website Design}

- Symmetrical design of the web page (Marcus, 2002, Callahan, 2006)

- Frequent use of photos of buildings, departments and the logo of university rather than people and students in their daily informal activities. (Marcus, 2002)

- Concentrating on prominent people (politically, religiously ,..) or professors as opposed to the students in the appearance and content of website (which conveys the sense of inequality and organizational hierarchy) (Marcus, 2002; Dormann and Chisalita, 2002)

- Focusing on religions and traditions with repeated use of religious and national symbols. (Marcus, 2002)

- Organizing large amount of information in a vertical or tall hierarchical order without attention to the importance and relationship of the information in their classification method, (just a mere long list of information is given). (Marcus, 2002)

- Structured/controlled access to the information with limited options. (Marcus, 2002)

- Error messages phrased in harsh tone rather than supporting and assisting messages. (Marcus, 2002)

- Noticeable/obvious and compulsory restrictions in the access of the information and security issues such as using IDs and passwords. (Marcus, 2002; Robbins\& Stylianou, 2002)

- Indicators of High Collectivism in Website Design

- Photos of elderly and experienced people as opposed to youth. (Marcus,2002; Rajkumar, 2003; Callahan 2006)

- Pictures and symbols of political, social or collective/group achievements in comparison with individual accomplishments. (Marcus, 2002; Rajkumar, 2003; Callahan 2006)

- Focusing on experiences traditions religions rather than new special issues. (Marcus ,2002)

- Using formal terminology in passive voice instead of direct and informal language in active voice. (Rajkumar, 2003)

- Lack of using argumentative and exaggerating phrases. (Marcus, 2002; Rajkumar, 2003)

- Posing organizational issues rather than market-oriented subjects. (Marcus, 2002)

- Restricted conventional access methods and options as opposed to a variety of customizable options such as enabling users to express their personal opinion. (Robbins and Stylianou, 2002) 
- Lack of respecting users' privacy in different ways for example enabling them to protect their privacy, to register in order to get access to some sections and to use secured connections. (Marcus, 2002)

\section{Indicators of High Femininity in Website Design}

- Demonstrating some tendencies towards family and relationship (quality of life) rather than competition and domination (quantity of life) for instance showing some symbols of money, possessions, and success, such as financial reports (Robbins \& Stylianou, 2002)

- Making use of feminine colors and pictures, for instance, showing students talking, studying and laughing together rather than masculine colors, photos and animations that express symbols of power and traditions, such as buildings (Marcus, 2002; Dormann \& Chisalita, 2002; Callahan 2006)

- Demonstrating some indications of social and cultural sensitivity (as a sign of paying attention to others) (Dormann \& Chisalita, 2002; Marcus, 2002)

- Focusing on details rather than providing a holistic view.

- Concentrating on attractive form of design rather than focusing on functionality and effectiveness of design. (Marcus, 2002)

- Variety of choices with an attitude towards a cooperative relationship and information exchange, as opposed to limited similar options leading towards certain goals and getting quick results (Marcus,2002)

- Organizing information based on social structures rather than business- and task-oriented views, with an executive attitude. (Marcus, 2002)

- Emphasizing on equality between genders and ignoring the distinction between them, e.g., showing hardworking ambitious women or modest sensitive men as opposed to emphasizing on gender distinction (making explicit different tones and expectations for each gender). (Marcus, 2002)

\section{Indicators of High Uncertainty Avoidance in Website Design}

- Making use of vertical design rather than horizontal design of web pages. (Marcus, 2002; Callahan 2006)

- Clear and understandable organization of the information. (Marcus, 2002)

- Providing users with different forms of clues to minimize ambiguity, such as using color codes and dedicated fonts. (Marcus, 2002)

- Limited amount of information with little scrolling rather than long pages with a lot of scrolling. . (Marcus, 2002)

- Simple navigation system with complete and precise user interactions, providing clear feedbacks regarding the status of the users, as well as providing clear help and the sitemap, as opposed to complicated navigation system with limited control that encourages users to surf and browse, open new tabs and windows, as well as abstract help and limited feedback (Marcus, 2002)

- Focus on rules, discipline and formalities rather than flexibility, informality, ambiguity and change. (Marcus, 2002)

- Simple and clear interface design of the website with consistent familiar objective and tangible symbols relating to people's daily lives as opposed to various unfamiliar abstract pictures and symbols. (Marcus, 2002)

\section{Methodology}

Conceptual model: Bearing in mind the afore mentioned cultural indicators of website design, the following conceptual model has been developed and later used as the basis for setting the questions of our questionnaire and analysis of the cultural aspects of website design. (Figure 1)

Materials and Methods: A methodological contribution of the present study is in using the user perception about the design features of the sample websites; in contrast, the majority of previous researchers used the 
content analysis method solely to examine the cultural elements and hence user communities were not involved in measuring and establishing their cultural preferences.

Research Method: We have used a two-phase research method for our research. Firstly, and not surprisingly, the work began with a concise literature review of the subject, and existing cultural indicators of website design were extracted, according to the models proposed by different researchers of the field. The results of this phase have been presented in the literature review section before in this paper. In the second phase, we sought advice from a number of academic experts in the field of sociology and IT to assess and reform the list of cultural indicators. Upon their review, some of indicators omitted, or changed and arranged to prepare the questionnaire as the means of survey.

Figure 1: Conceptual Framework based upon Cultural indicators of Website Design

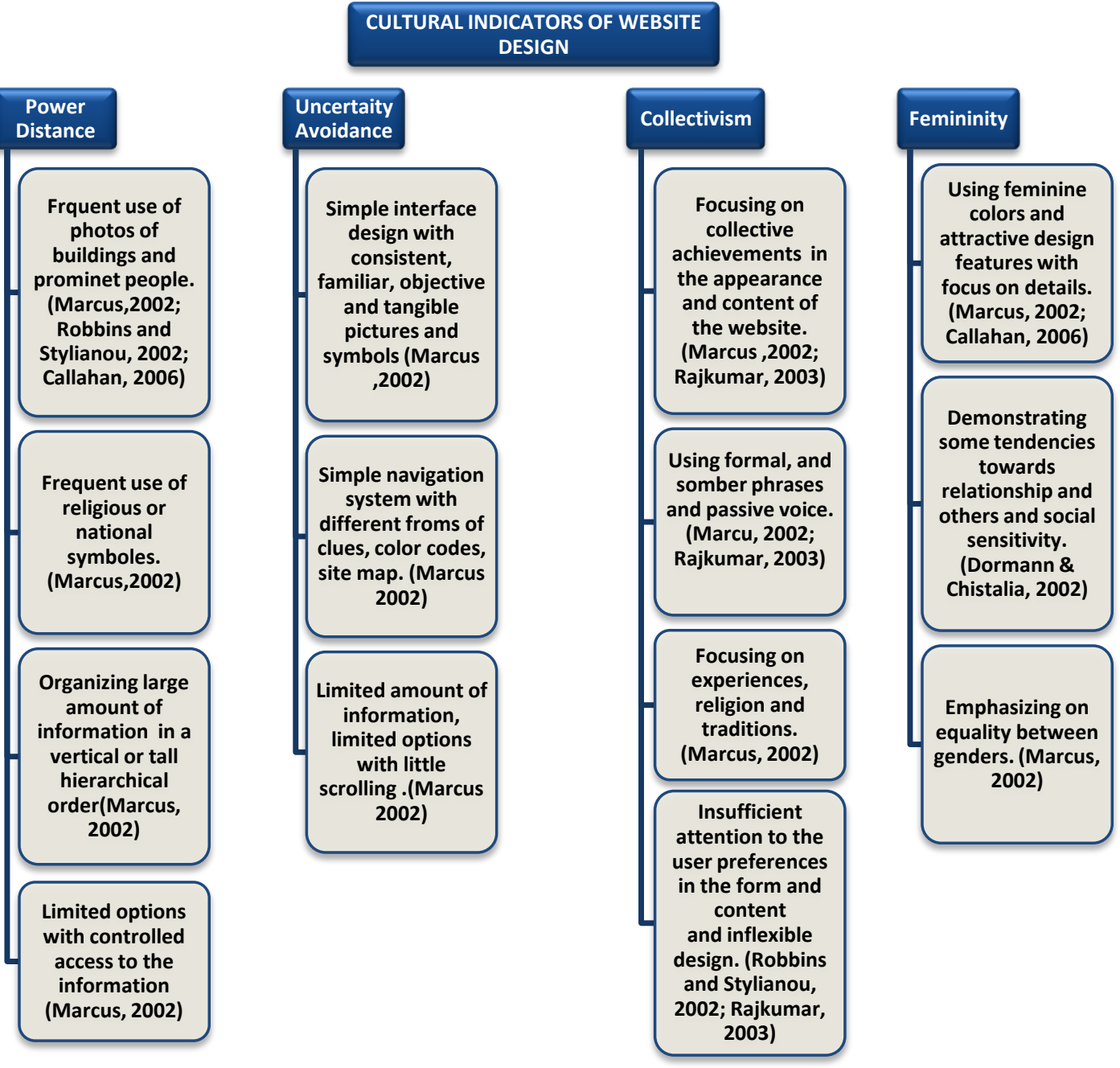

Questionnaire: The questionnaire as means of survey consists of two major groups of questions. The participants were asked to refer to the website and answer the questions in the questionnaire according to a five point scale (sorted from in all cases to none of the cases) The first group regarded graphical and visual features, terminology, organization of information, and proper navigation of the website. This set of questions intended to measure how users describe the design elements of website for instance "To what extent did you see the students in their daily life in the photos of the website?" or "Did you find any noticeable restrictions in the access of information?" or "Is the information organized based on task-oriented views with an executive attitude in the website? (For example administrative vice president, vice president for academic or students 
affairs)". The second group of questions was about the user perception (feeling, ideas, and impression) about the website design for example "How understandable and clear were the design and organization of information to you?" or "How much attention do you think is given to the attractiveness of the design in this website?" This composition of questions enables researchers to describe features of the website, and to compare the preferences and impressions of the users with regard to the Hofstede model of national cultural dimensions.

Sample and analytical method: As universities websites as well as other educational institutions are regarded to be social and cultural institutions, according to Callahan (2006), they can be considered as an ideal genre for identifying cultural differences. Rajkumar (2003) mentioned that cultural values should be reflected in the goals and objectives of universities, therefore their cultural preferences should be shown in the design of their web page. To examine this fact, three old-established Iranian state universities have been chosen as sample of study namely 'University of Tehran', 'Sharif University of Technology' and 'Shahid Beheshti University'. A group of 25 students were contacted via email. We asked them to refer to the website and answer the questions in the questionnaire according to a five-point scale (sorted from in all cases to none of the cases). Before answering the questions, the participants were asked to complete a specified task and find a particular piece of information in each website. For example, they were asked to find the tuition fee of $\mathrm{PhD}$ program or the minimum undergraduate cumulative grade point average for admission to the master program. The task was given to the participants to make sure that they have visited and searched the website and they are familiar enough with its design to answer our questions. Average score of websites given by the participants in each cultural dimension of Hofstede model, has been compound ed separately. As the sample size was small in number, we firstly tested the normality of the distribution of data, by Colmogorov-Smoirnov test in SPSS. Then, one sample T-test was conducted separately for each cultural dimension to compare the average score of websites with that of Hofstede's model for Iran. The results are articulated in the following section.

\section{Results}

After analyzing the data of our questionnaires, the average scores given by the users to the sample group of Iranian university websites for each cultural dimension were as follows :( Table1)

Table 1: Summary of websites' scores in each cultural dimension based on users' opinion

\begin{tabular}{lllll}
\hline & N & Mean & Std. Deviation & Std. Error Mean \\
\hline UNCERTAINTY AVOIDANCE & 25 & 2.80 & .44 & .089 \\
POWER DISTANCE & 25 & 3.24 & .21 & .042 \\
FEMINITY & 25 & 2.28 & .32 & .064 \\
COLLECTIVISM & 25 & 3.41 & .23 & .047 \\
\hline
\end{tabular}

The average score of websites in uncertainty avoidance was 2.80 or $\% 56(S D=.44)$.

The average score of websites in power distance was 3.24 or \%64.8 $(S D=.21)$.

The average score of websites in femininity was 2.28 or $\% 45.6(S D=.32)$.

The average score of websites in collectivism was 3.41 or $\% 68.2(S D=.23)$.

Hence, according to the perception of the end users, Iranian university websites have collectivism and power distance as their highest Hofstede dimension at 68 and 64 respectively and femininity as their lowest ranking at 45 . Then, one sample t-test was conducted to compare the websites' score and the rankings of Iran in theory of Hofstede. There was a significant difference in power distance score of websites with the score of Iran in power distance at 58 in theory of Hofstede $(t(25)=7.92, p<.005)$. There were also a meaningful difference in collectivism $(t(25)=9.77, p<.005)$ and femininity $(t(25)=-8.63, p<.005)$ scores of websites with rankings of Iran in Hofstede model. However, regarding uncertainty avoidance, the result did not reveal a significant difference between websites score and that of Hofstede model in uncertainty avoidance dimension $(t(25)=-1.62, p>.005)$. (Table 2$)$ 
Table 2: One Sample Test Result

\begin{tabular}{llll}
\hline & t & Sig. (2-tailed) & Test Value \\
\hline UNCERTAINTY AVOIDANCE & -1.623 & .118 & 2.95 \\
POWER DISTANCE & 7.921 & .000 & 2.9 \\
COLLECTIVISM & 9.778 & .000 & 2.95 \\
FEMINITY & -8.639 & .000 & 2.85 \\
\hline
\end{tabular}

The chart below compares the cultural attributes of Iranian university websites and characteristics of Iranian culture in Hofstede model. (Figure 2)

\section{Figure 2: Comparison of Cultural Characteristics of Iranian University Websites with the Hofstede} Model

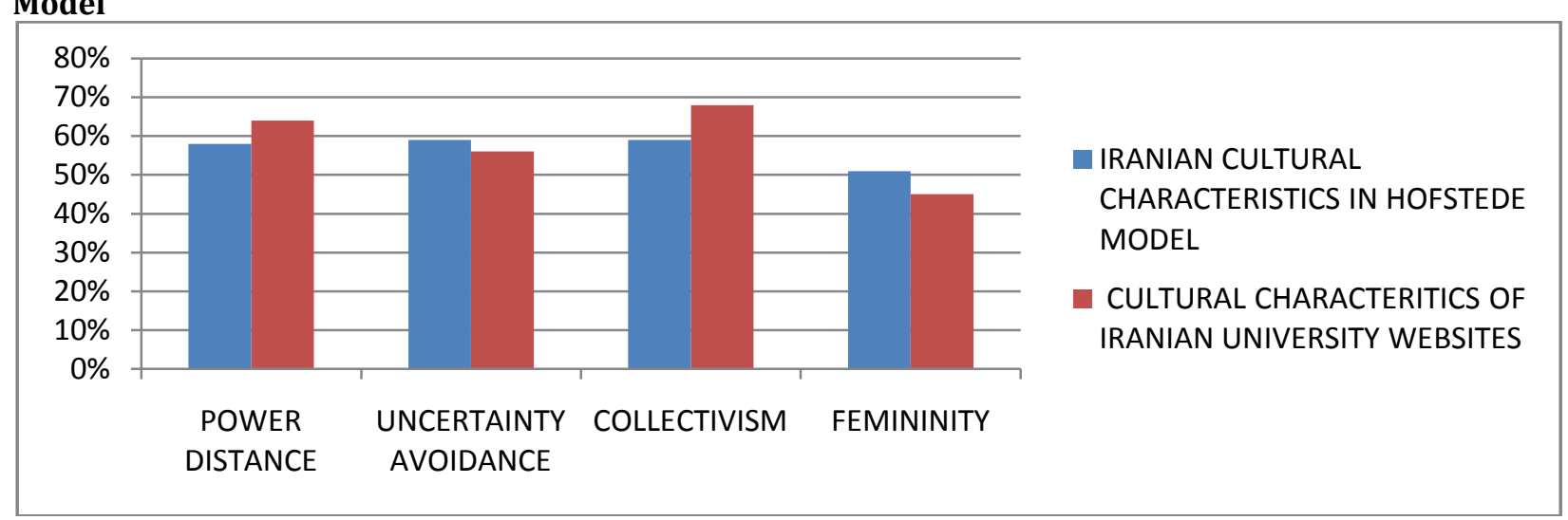

\section{Discussion and Conclusion}

As it is suggested in cultural studies of interface design, it is important for websites (or other forms of interface, for that matter) to reflect the cultural characteristics and values of their target users, so that the users establish an effective communication with the website and feel comfortable and satisfied with their experience. Based on our findings, the design of Iranian university websites portrays a collectivist culture with a relatively high characteristic of power distance, masculine and an average degree of uncertainty avoidance. Matching up these findings with the cultural rankings of Hofstede, the design of Iranian university websites is significantly inconsistent with Hofstded's theory and they do not reflect their culture in three of the four cultural dimensions namely individualism, power distance and masculinity. The website designs represent a more collectivistic and masculine culture with higher indices of power distance, than what is specified in Hofstede's model. However, the design of the websites seems to be compatible with their local culture concerning the uncertainty avoidance attribute. It is worth mentioning that some scholars in the field of sociology and management have challenged the rankings of Iran in the theory of Hofstede and believe that his cultural model about Iran needs to be reassessed particularly in relation to different subcultures in Iran. Therefore, we verified the Hofstede model using a set of questions that this mismatch between cultural characteristics and website design indeed leads to unpleasant user experience. We have noticed that the vast majority (nearly all) of the participants, regardless of their subcultures, stated that they did not enjoy their experience with their websites and they found the design too formal, rigid, non-creative and unpleasant. Therefore, a need to change towards more individualistic and elegant and less formal design is indicated according to both the Hofstede theory and the perception of the end users. Based on the results of the present study, we suggest that Iranian websites need to harmonize their design with the culture of their target users in different aspects. Regarding collectivism, power distance and femininity that revealed significant difference in our study, they need to establish more individualistic and less formal design features such as using photos of people and students in their daily informal activities rather than buildings or students formal occasions, direct and informal language in active voice, creative graphical design rather than repetitive boring form of design, demonstrating more individual accomplishments, interests and developing more 
customizable features. In order to improve the femininity aspects, it seems imperative to reflect relations that are more informal and pay more attention to details and particulars. However, with respect to uncertainty avoidance, neither the result of the comparison with Hofstede model, nor the viewpoint of participants in the questionnaire showed meaningful inconsistency with the culture and impression of the Iranian sample group of users. The users gave mostly intermediate score to the uncertainty avoidance aspect, which is consistent with Hofstede's model.

\section{References}

Ahmed, T., Mouratidis, H. \& Preston, D. (2008). Website Design and Localization: A Comparison of Malaysia and Britain. International Journal of Cyber Society and Education, 1(1) 3-16.

Alostath, J. M. \& Khalfan, A. M. (2007). Cross-Use: Cross-Cultural Usability User Evaluation-In-Context. The 2nd international conference on Usability and internationalization, 225-234, Beijing, China

Callahan, E. (2006). Cultural Similarities and Differences in the Design of University Web sites. Journal of Computer-Mediated Communication, 11, 239-273,

Cyr, D. (2008). Modeling Web site design across cultures: Relationships to trust, satisfaction, and e-loyalty. Journal of Management Information Systems, 24(4) 47-72

Dormann, C. \& Chisalita, C. (2002). Cultural values in web site design. The Eleventh European Conference on Cognitive Ergonomics, 179-186, Catania, Italy.

Hofstede, G. (1991). Cultures and organizations: Software of the mind. London: McGraw-Hill.

Lodge, C. (2007). The Impact of Culture on Usability: Designing Usable Products for the International User. The 2nd international conference on Usability and Internationalization, HCI and Culture, 365-368, Beijing, China.

Marcus, A. (2001). Cross-Cultural User-Interface Design. Human-Computer Interface International Conference, 502-505, New Orleans, USA.

Marcus, A. (2002). Mapping User-Interface Design to Culture Dimensions. Workshop of CHI, SIGCHI Annual Conference, 21-24, Minneapolis, USA.

Marcus, A. \& Gould, E. (2000). Crosscurrents: cultural dimensions and global Web user-interface design. Interactions, 7(4), 32-46.

Marcus, A. \& Hamoodi, G. (2009). The Impact of Culture on the Design of Arabic Websites. International Conference of Human Computer Interaction, 386 - 394, San Diego, USA

Nantel, G. (2008). The impact of language and culture on perceived websites usability. Journal of Engineering and Technology Management, 25, 112-122.

Rajkumar, S. (2003). University Web sites: Design differences and reflections of culture. Unpublished manuscript, School of Library and Information Science, Indiana University Bloomington

Robbins, S. \& Stylianou, A. C. (2002). A study of cultural differences in global corporate web sites. Journal of Computer Information Systems, 42, 3-9

Robbins, S. S. \& Stylianou. (2008). A longitudinal study of cultural differences in global corporate web sites, Journal of International Business and Cultural Studies, 3, 178-184.

Van-Iwaarden, J., van-der-Wiele, T., Ball, L. \& Millen, R. (2004). Perceptions about the quality of web sites: a survey amongst students at Northeastern University and Erasmus University. Information and Management, 41(8), 947-959 